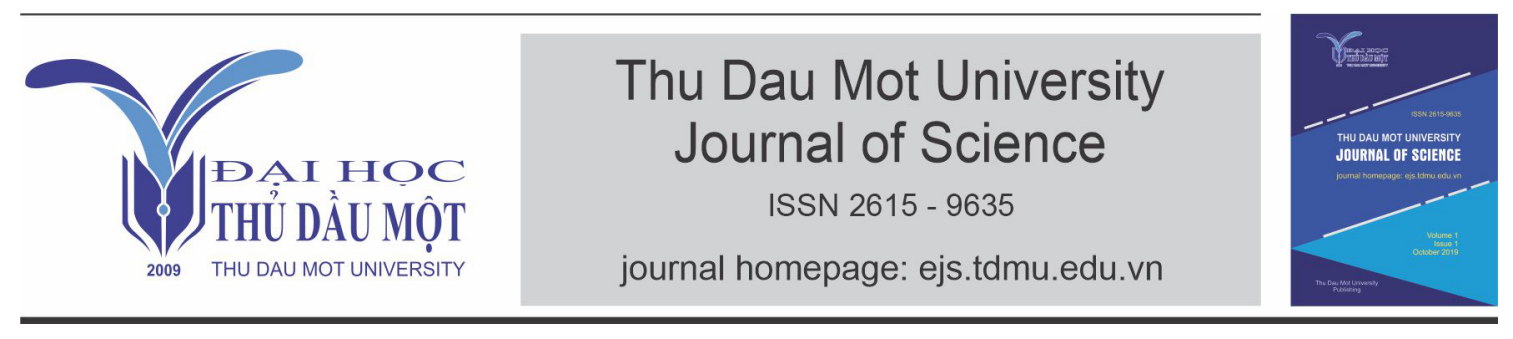

\title{
On the lower semicontinuity of the solution map- ping for parametric vector mixed quasivariational inequality problem of the Minty type
}

by Nguyen Van Hung (Posts and Telecommunications Institute of Technology Ho Chi Minh City), Vo Viet Tri (Thu Dau Mot university)

Article Info: Received 10 April. 2020, Accepted 7 May. 2020, Available online 30 May. 2020

Corresponding author: trivv@tdmu.edu.vn (Vo Viet Tri)

https://doi.org/10.37550/tdmu.EJS/2020.02.041

\begin{abstract}
In this paper, we study a class of parametric vector mixed quasivariational inequality problem of the Minty type (in short, (MQVIP)). Afterward, we establish some sufficient conditions for the stability properties such as the inner-openness, lower semicontinuity and Hausdorff lower semicontinuity of the solution mapping for this problem. The results presented in this paper is new and wide to the corresponding results in the literature
\end{abstract}

Keywords: problem of the minty type, inner-openness, lower semicontinuity, Hausdorff lower semicontinuity

\section{Introduction}

In 1980, Giannessi [3] was introduced a vector variational inequality in a finitedimensional Euclidean space. After that, there are many papers on the property of solution sets for vector variational inequality problems in abstract spaces, see $[1,8,4]$ and the references therein. On the other hand, stability of solutions for 
parametric vector mixed quasivariational inequality problem is an important topic in optimization theory and applications. Recently, the continuity, especially the upper semicontinuity, lower semicontinuity and Hausdorff lower semicontinuity of the solution sets for parametric optimization problems, parametric vector variational inequality problems and parametric vector quasiequilibrium problems have been studied in the literature, see $[1,4,5,6,10]$.

Motivated and inspired by the works mentioned above, in this paper we introduce and study the parametric vector mixed quasivariational inequality problem. Let $X, Y$ be two Hausdorff topological vector spaces, $P$ be a closed pointed convex in $Y$ with int $P \neq \emptyset, A$ be a nonempty subset of $X$ and $\Lambda$ be a topological space. The space of all linear continuous operators from $X$ into $Y$ denoted by $L(X, Y)$. Let $K: A \times \Lambda \rightrightarrows X, T: A \times \Lambda \rightrightarrows L(X, Y)$ be set-valued mappings, and let $g: A \times A \times \Lambda \rightarrow X, f: A \times A \times \Lambda \rightarrow Y$ be two continuous single-valued mappings. Denoted $\langle z, x\rangle$ by the value of a linear operator $z \in L(X, Y)$ at $x \in X$. We always assume that $\langle.,$.$\rangle is continuous.$

For $\gamma \in \Lambda$, we consider the following parametric vector mixed quasivariational inequality problems (in short, (MQVIP-j), $\mathrm{j}=1,2$ ).

(MQVIP-1) Find $\bar{x} \in A$ such that

$$
\left\{\begin{array}{l}
\bar{x} \in K(\bar{x}, \gamma) \\
\langle z, g(y, \bar{x}, \gamma)\rangle+f(y, \bar{x}, \gamma) \notin-i n t P \text { for all } y \in K(\bar{x}, \gamma), z \in T(y, \gamma)
\end{array}\right.
$$

(MQVIP-2) Find $\bar{x} \in A$ such that

$$
\left\{\begin{array}{l}
\bar{x} \in K(\bar{x}, \gamma) \\
\langle z, g(y, \bar{x}, \gamma)\rangle+f(y, \bar{x}, \gamma) \notin-P \text { for all } y \in K(\bar{x}, \gamma), z \in T(y, \gamma)
\end{array}\right.
$$

For each $\gamma \in \Lambda$ we let $E(\gamma):=\{x \in X \mid x \in K(x, \gamma)\}$ and $\Psi_{j}: \Lambda \rightrightarrows X$ be a setvalued mapping such that $\Psi_{j}(\gamma)$ is the solution set of (MQVIP-j), j=1,2. Throughout this paper, we always assume that $\Psi_{j}(\gamma) \neq \emptyset$ for each $\gamma$ in the neighborhood of $\gamma_{0} \in \Lambda$.

The structure of our paper is as follows. In the first part of this article, we introduce the model parametric vector mixed quasivariational inequality problems of the Minty type. Section 2, we recall definitions for later uses. In Section 3, we establish the lower semicontinuity, inner-openness and Hausdorff lower semicontinuity of the solutions for parametric vector mixed quasivariational inequality problem of the Minty type. 


\section{Preliminaries}

In this section, we recall some basic definitions and their some properties.

We first recall a new limit in [7,9], the inferior open limit. Let $X$ and $Y$ be two topological vector spaces.

Definition 2.1 (see $[7,9]$ ) Let $A$ be a nonempty subset of $X$ and $G: A \rightrightarrows Y$ be a multifunction.

(i) For $x_{0} \in A$, denote liminfo $x_{x \rightarrow x_{0}} G(x):=\{z \in Y$ : there are open neighborhoods $U$ of $x_{0}$ and $V$ of $z$ such that $V \subset G(x)$ for all $\left.x \in U \backslash\left\{x_{0}\right\}\right\}$.

(ii) $\operatorname{Limsup}_{x \rightarrow x_{0}} G(x):=\left\{y \in Y\right.$ : there exists a net $\left\{\left(x_{\alpha}, y_{\alpha}\right)\right\} \subset A \times Y$ converging to $\left(x_{0}, y\right)$ with $\left.y_{\alpha} \in G\left(x_{\alpha}\right)\right\}$

(iii) $G$ is said that inner-open at $x_{0}$ if $\operatorname{liminfo}_{x \rightarrow x_{0}} G(x) \supset G\left(x_{0}\right) . G$ is said to be inner-open on $A$ if it is inner-open at every $a \in A$.

From the Item (iii) of Lemma 2.1 in [7], we deduce that $\operatorname{liminfo}_{x \rightarrow x_{0}} G(x)=$ $\left[\lim \sup _{x \rightarrow x_{0}} G^{c}(x)\right]^{c}$, where $G^{c}(x)=Y \backslash G(x)$.

Definition 2.2 ([2]). Let $X$ and $Y$ be two topological vector spaces and $G: A \subset$ $X \rightrightarrows Y$ be a multifunction.

(i) $G$ is said to be lower semicontinuous (lsc) at $x_{0} \in A$ if for every open subset $U$ of $Y$ with $G\left(x_{0}\right) \cap U \neq \emptyset$, there exists a neighborhood $N$ of $x_{0}$ such that $G(x) \cap U \neq \emptyset$, for all $x \in N \cap A$.

(ii) $G$ is said to be upper semicontinuous (usc) at $x_{0} \in A$ if for each open set $U \supset G\left(x_{0}\right)$, there is a neighborhood $N$ of $x_{0}$ such that $U \supset G(x)$ for all $x \in N \cap A$.

(iii) $G$ is said to be Hausdorff lower semicontinuous (H-lsc) at $x_{0} \in A$ if for each neighborhood $B$ of the origin in $Y$, there exists a neighborhood $N$ of $x_{0}$ such that $G\left(x_{0}\right) \subset G(x)+B$ for all $x \in N \cap A$.

(iv) $G$ is said to be closed at $x_{0} \in A$ if and only if for every net $\left\{\left(x_{\alpha}, y_{\alpha}\right)\right\} \subset A \times Y$ converging $\left(x_{0}, y_{0}\right)$ with $y_{\alpha} \in G\left(x_{\alpha}\right)$, we have $y_{0} \in G\left(x_{0}\right)$. 
(v) $G$ is said to be lower semicontinuous (upper semicontinuous, Hausdorff lower semicontinous, closed, respectively) on $A$ if it is lower semicontinuous (upper semicontinuous, Hausdorff lower semicontinous, closed, respectively) at each $x_{0} \in A$.

Lemma 2.3 ([2]). Let $X$ and $Y$ be two topological vector spaces and $G: A \subset X \rightrightarrows$ $Y$ be a multifunction. Then, the following assertions hold.

(i) $G$ is lsc at $x_{0} \in A$ iff for every $y \in G\left(x_{0}\right)$ and each neighborhood $V$ of $y$, there exists a neighborhood $U$ of $x_{0}$ such that $G(x) \cap V \neq \emptyset$ for all $x \in U \cap A$.

(ii) If $G$ is $H$-lsc at $x_{0}$ then $G$ is lsc at $x_{0}$. The converse is true if $G\left(x_{0}\right)$ is compact,

(iii) If $G$ has compact values, then $G$ is usc at $x_{0}$ if and only if, for each net $\left\{x_{\alpha}\right\} \subset X$ which converges to $x_{0}$ and for each net $\left\{y_{\alpha}\right\}$ with $y_{\alpha} \in G\left(x_{\alpha}\right)$, there are $y \in G\left(x_{0}\right)$ and a subnet $\left\{y_{\beta}\right\}$ of $\left\{y_{\alpha}\right\}$ such that $y_{\beta} \rightarrow y$.

\section{$3 \quad$ Main Results}

In this section, with the contexts and hypothesis of the problem (MQVIP-j) describing in the Section 1 we establish the inner-openness, lower semicontinuity and Hausdorff lower semicontinuity of the solution set of (MQVIP-j).

Theorem 3.1 Suppose the following conditions hold

(i) E is inner-open on $\Lambda$,

(ii) $K$ is upper semicontinuous on $A \times \Lambda$ with compact values, and

(iii) $T$ is upper semicontinuous on $A \times \Lambda$ with compact values.

Then $\Psi_{2}$ is inner-open on $\Lambda$.

Proof. Suppose to the contrary that $\Psi_{2}$ is not inner-open at $\gamma_{0}$. Then, there exists $x_{0} \in \Psi_{2}\left(\gamma_{0}\right), x_{0} \notin \operatorname{liminfo}_{\gamma \rightarrow \gamma_{0}} \Psi_{2}(\gamma)$. Since

$$
\operatorname{liminfo}_{\gamma \rightarrow \gamma_{0}} \Psi_{2}(\gamma)=\left[\limsup _{\gamma \rightarrow \gamma_{0}}\left(\Psi_{2}\right)^{c}(\gamma)\right]^{c},
$$

we have $x_{0} \in \lim \sup _{\gamma \rightarrow \gamma_{0}}\left(\Psi_{2}\right)^{c}(\gamma)$. Thus, there exists a net $\left\{\left(\gamma_{\alpha}, x_{\alpha}\right)\right\}$ converging to $\left(\gamma_{0}, x_{0}\right)$ with $x_{\alpha} \in\left(\Psi_{2}\right)^{c}\left(\gamma_{\alpha}\right)$. Since $E$ is inner-open at $\gamma_{0}$ and $x_{0} \in E\left(\gamma_{0}\right)$ which 
implies $x_{0} \in \operatorname{liminfo}_{\gamma \rightarrow \gamma_{0}} E(\gamma)$. There exist neighborhoods $U$ of $\gamma_{0}, N$ of $x_{0}$ such that $x_{0} \in N \subset E(\gamma)$ for all $\gamma \in U$. Since $\left(x_{\alpha}, \gamma_{\alpha}\right) \rightarrow\left(x_{0}, \gamma_{0}\right)$ and by the above contradiction assumption, there must be a subnet $\left\{\left(x_{\beta}, \gamma_{\beta}\right)\right\}$ of net $\left\{\left(x_{\alpha}, \gamma_{\alpha}\right)\right\}$ such that for all $\beta, x_{\beta} \notin \Psi_{2}\left(\gamma_{\beta}\right)$, i.e., there exist $y_{\beta} \in K\left(x_{\beta}, \gamma_{\beta}\right)$ and $z_{\beta} \in T\left(y_{\beta}, \gamma_{\beta}\right)$ such that

$$
\left\langle z_{\beta}, g\left(y_{\beta}, x_{\beta}, \gamma_{\beta}\right)\right\rangle+f\left(y_{\beta}, x_{\beta}, \gamma_{\beta}\right) \in-P .
$$

Since $K$ is upper semicontinuity on $A \times \Lambda$ and $K\left(x_{0}, \gamma_{0}\right)$ is compact, one has $y_{0} \in K\left(x_{0}, \gamma_{0}\right)$ such that $y_{\beta} \rightarrow y_{0}$ (taking a subnet if necessary) by Lemma 2.3. Also, by the upper semicontinuity of $T$ at $\left(y_{0}, \mu_{0}\right)$ and $T\left(y_{0}, \gamma_{0}\right)$ is compact, one has $z_{\beta}^{\prime} \in T\left(y_{\beta}, \gamma_{\beta}\right)$ such that $z_{\beta}^{\prime} \rightarrow z_{0}$. Since $g, f$ and $\langle.,$.$\rangle are continuous, we have$

$$
\left\langle z_{\beta}^{\prime}, g\left(y_{\beta}, x_{\beta}, \gamma_{\alpha}\right)\right\rangle+f\left(y_{\beta}, x_{\beta}, \gamma_{\beta}\right) \rightarrow\left\langle z_{0}, g\left(y_{0}, x_{0}, \gamma_{0}\right)\right\rangle+f\left(y_{0}, x_{0}, \gamma_{0}\right) .
$$

From $P$ is closed, (3.1) and (3.2), it follows that

$$
\left\langle z_{0}, g\left(y_{0}, x_{0}, \gamma_{0}\right)\right\rangle+f\left(y_{0}, x_{0}, \gamma_{0}\right) \in-P,
$$

which is impossible since $x_{0} \in \Psi_{2}\left(\gamma_{0}\right)$. The roof is complete.

The following example shows that the inner-openness of $E$ is essential.

Example 3.1 Let $X=Y=\mathbb{R}, A=[0,1], \Lambda=[0,1], P=\mathbb{R}_{+}$, and for every $(x, y, \gamma) \in A \times A \times \Lambda$ we define $f(y, x, \gamma)=0$;

$$
\begin{aligned}
K(x, \gamma) & = \begin{cases}(0,1] & \text { if } \gamma \in(0,1], \\
{[-1,0]} & \text { if } \gamma=0 ;\end{cases} \\
T(y, \gamma) & =\left[1,2^{1+\cos ^{2} \gamma}\right] \text { and } \\
g(y, x, \gamma) & =\left\{2+3 \gamma^{2}\right\} .
\end{aligned}
$$

It follows that $E(\gamma)=(0,1]$ for all $\gamma \in(0,1]$ and $E(0)=[-1,0]$. It is not hard to see that the assumptions (ii) and (iii) in Theorem 3.1 are satisfied. But $E$ is not inner-open at 0 . Hence, $\Psi_{2}$ also not inner-open at 0 . Thus, Theorem 3.1 cannot be applied. In fact, $\Psi_{2}(0)=[-1,0]$ and $\Psi_{2}(\gamma)=(0,1]$ for all $\gamma \in(0,1]$.

Next, we establish the lower semicontinuity of the solutions for parametric vector mixed quasivariational inequality problem of the Minty type (MQVIP-1). We denote

$$
\begin{aligned}
\operatorname{Er}\left(\Psi_{1}\right) & =\{(x, \gamma) \in A \times \Gamma:<z, g(y, x, \gamma)>+f(y, x, \gamma) \notin-\operatorname{int} P \\
& \forall y \in K(x, \gamma) \text { and } z \in T(y, \gamma)\} \\
\operatorname{Graph}\left(\Psi_{1}\right) & =\left\{(x, \gamma) \in A \times \Gamma: x \in \Psi_{1}(\gamma)\right\} .
\end{aligned}
$$


Theorem 3.2 Suppose the following conditions hold:

(i) $E$ is lower semicontinuous on $\Lambda$;

(ii) A net $\left(x_{\alpha}, \gamma_{\alpha}\right)$ converging to some element in $\operatorname{Graph}\left(\Psi_{1}\right)$ implies that there exists $\alpha$ satisfying $\left(x_{\alpha}, \gamma_{\alpha}\right) \in \operatorname{Er}\left(\Psi_{1}\right)$.

Then, $\Psi_{1}$ is lower semicontinuous on $\Lambda$.

Proof. Suppose to the contrary that $\Psi_{1}$ is not lower semicontinuous at $\gamma_{0}$, by Lemma 2.3 there exist $x_{0} \in \Psi_{1}\left(\gamma_{0}\right)$ and a neighborhood $V$ of $x_{0}$ such that

for every neighborhood $U$ of $\gamma_{0}$, we have $\Psi_{1}\left(\gamma_{0}\right) \cap V=\emptyset$ for all $\gamma \in U$.

This, we can find a net $\gamma_{\alpha}$ such that $\gamma_{\alpha} \rightarrow \gamma_{0}$

$$
\Psi_{1}\left(\gamma_{\alpha}\right) \cap V=\emptyset \forall \alpha
$$

Since $E$ is lower semicontinuous at $\gamma_{0} \in \Lambda$, there is a net $\left\{x_{\alpha}\right\}$ with $x_{\alpha} \in E\left(\gamma_{\alpha}\right)$, $x_{\alpha} \rightarrow x_{0}$. Without loss of generality we can assume $x_{\alpha} \in V$ for al $\alpha$ (taking a subnet if necessary). By the condition (ii), there exists $\alpha$ satisfying $\left(x_{\alpha}, \gamma_{\alpha}\right) \in \operatorname{gr}\left(\Psi_{1}\right)$, thus $\left(x_{\alpha}, \gamma_{\alpha}\right) \in \operatorname{Graph}\left(\Psi_{1}\right)$ which contradicts (3.4). Therefore, $\Psi_{1}$ is lower semicontinous.

The following example ensures that the lower semicontinuity of $E$ in Theorem 3.2 is essential.

Example 3.2 Let $X=Y=\mathbb{R}, A=[-1,1], P=\mathbb{R}_{+}, \Lambda=[0,1]$, for $(y, x, \gamma) \in$ $A \times A \times \Lambda$ we defined

$$
\begin{aligned}
& T(y, \gamma)=[0,1], f(y, x, \gamma)=g(y, x, \gamma)=\left\{1+2^{\sin ^{2}(\gamma)}\right\} \\
& K(x, \gamma)= \begin{cases}\{-1,0\}, & \text { if } \gamma=0, \\
\{0,1\}, & \text { if } \gamma \in(0,1] .\end{cases}
\end{aligned}
$$

It is clear to see that $E(0)=\{-1,0\}, E(\gamma)=\{0,1\}, \forall \gamma \in(0,1]$ and the assumption (ii) of Theorem 3.2 is fulfilled. However, $\Psi_{1}$ is not lower semicontinuous at 0 . The reason is that $E$ is not lower semicontinuous at 0 .

Finally, we study Hausdorff lower semicontinuity of the solutions for parametric vector mixed quasivariational inequality problem of the Minty type (MQVIP-1). 
Theorem 3.3 Impose the assumption of Theorem 3.2 and the following additional condition:

(iii) $K\left(., \gamma_{0}\right)$ and $T\left(., \gamma_{0}\right)$ are lower semicontinuous on $X$ and $E\left(\gamma_{0}\right)$ is compact.

Then $\Psi_{1}$ is Hausdorff lower semicontinuous on $\Lambda$.

Proof. We first prove that $\Psi_{1}\left(\gamma_{0}\right)$ is closed. Indeed, we let $\left\{x_{\alpha}\right\} \subset \Psi_{1}\left(\gamma_{0}\right)$ such that $x_{\alpha} \rightarrow x_{0}$. If $x_{0} \notin \Psi_{1}\left(\gamma_{0}\right)$, then there exist $y_{0} \in K\left(x_{0}, \gamma_{0}\right)$ and $z_{0} \in T\left(y_{0}, \gamma_{0}\right)$ such that

$$
\left\langle z_{0}, g\left(y_{0}, x_{0}, \gamma_{0}\right)\right\rangle+f\left(y_{0}, x_{0}, \gamma_{0}\right) \in-\operatorname{int} P .
$$

From the lower semicontinuity of $K\left(., \gamma_{0}\right)$ at $x_{0}$, by the Lemma 2.3 one has a net $\left\{y_{\alpha}\right\}$ converging to $y_{0}$ with $y_{\alpha} \in K\left(x_{\alpha}, \gamma_{0}\right)$. Also, by the lower semicontinuity of $T\left(., \gamma_{0}\right)$ at $y_{0}$, one has a net $z_{\alpha} \rightarrow z_{0}$ with $z_{\alpha} \in T\left(y_{\alpha}, \gamma_{0}\right)$. Since $\left\{x_{\alpha}\right\} \subset \Psi_{1}\left(\gamma_{0}\right)$, we have

$$
\left\langle z_{\alpha}, g\left(y_{\alpha}, x_{\alpha}, \gamma_{0}\right)\right\rangle+f\left(y_{\alpha}, x_{\alpha}, \gamma_{0}\right) \notin-\operatorname{int} P \text { for all } \alpha \text {. }
$$

Since $\left(x_{\alpha}, z_{\alpha}, y_{\alpha}\right) \rightarrow\left(x_{0}, z_{0}, y_{0}\right)$ and from the continuity of $g, f,\langle.,$.$\rangle and (3.6), we$ have

$$
\left\langle z_{0}, g\left(y_{0}, x_{0}, \gamma_{0}\right)\right\rangle+f\left(y_{0}, x_{0}, \gamma_{0}\right) \notin-\operatorname{int} P
$$

we see a contradiction between (3.5) and (3.7). Hence, $\Psi_{1}\left(\gamma_{0}\right)$ is a closed set.

On the other hand, $\Psi_{1}\left(\gamma_{0}\right)$ is closed subset of the compact $E\left(\gamma_{0}\right)$, therfore, it is compact. Since $\Psi_{1}$ is lower semicontinuous in $\Lambda$ (by Theorem 3.2) and $\Psi_{1}\left(\gamma_{0}\right)$ is compact, by Lemma 2.3, it follows that $\Psi_{1}$ is Hausdorff lower semicontinuous in $\Lambda$.

Remark 3.1 If $A=X=\mathbb{R}^{m}, Y=\mathbb{R}, P=\mathbb{R}_{+}, g(y, x, \gamma)=y-x, f(y, x, \gamma)=0$, then the problem (MQVIP-1) is reduced to the following quasivariational inequality problem of the Minty type (in short, (MVI)):

(MVI) Find $\bar{x} \in X$ with $\bar{x} \in K(\bar{x}, \gamma)$ such that

$$
\langle\bar{z}, y-\bar{x}\rangle \geq 0, \forall y \in K(\bar{x}, \gamma), z \in T(y, \gamma) .
$$

This problem has been studied in [8]. Then the lower semicontinuity of the solution set in Theorem 4.1 in [8] is a particular case of our Theorem 3.2, while our Theorem 3.3 is completely different Theorem 4.4 in [8]. Note that, our Theorem 3.1 is new for vector mixed quasivariational inequality problem of the Minty type. 


\section{Acknowledgements}

The authors wish to thank the anonymous referees for their valuable comments. This research is funded by Thu Dau Mot University, Binh Duong province, Viet Nam.

\section{References}

[1] L.Q. Anh, N.V. Hung, (2018). Gap functions and Hausdorff continuity of solution mappings to parametric strong vector quasiequilibrium problems, J. Ind. Manag. Optim., 14, 65-79.

[2] J.P. Aubin, I. Ekeland, (1984). Applied Nonlinear Analysis, John Wiley and Sons, New York.

[3] F. Giannessi, (1980). Theorems of alternative, quadratic programmes and complementarity problems, in: R. W. Cottle, F. Giannessi, J. L. Lions (Eds.), Variational Inequalities and Complementarity Problems, Wiley, Chichester, 151-186.

[4] N.V. Hung, (2013). Stability of a solution set for parametric generalized vector mixed castration inequality problem, J. Inequal. Appl., 276, 1-13.

[5] N.V. Hung, (2018). On the stability of the solution mapping for parametric traffic network problems, Indag. Math., 29, 885-894.

[6] N.V. Hung, N.M. Hai, (2019). Stability of approximating solutions to parametric bilevel vector equilibrium problems and applications, Comput. Appl. Math., $38,1-17$.

[7] P.Q. Khanh, D.T. Luc, (2008). Stability of solutions in parametric variational relation problems, Set-Valued Anal., 16, 1015-1035.

[8] C.S. Lalitha, G. Bhatia, (2011). Stability of parametric quasivariational inequality of the Minty type, J. Optim. Theory Appl., 148 , 281-300.

[9] R.T. Rockafellar, R. J-B. Wets, (1998). Variational Analysis, Springer, Berlin Heidelberg, .

[10] J. Zhao, (1997). The lower semicontinuity of optimal solution sets, J. Math. Anal. Appl., 207, 240-254. 